\title{
O333 Treatment discontinuation and virological failure amongst HIV-positive individuals starting second-line combination antiretroviral therapy (CART) CJ Smith*1, FC Lampe ${ }^{1}$, M Youle ${ }^{2}$, MA Johnson ${ }^{2}$ and AN Phillips ${ }^{1}$
}

Address: ${ }^{1}$ University College Medical School, London, UK and ${ }^{2}$ Royal Free Hospital, London, UK

* Corresponding author

from Ninth International Congress on Drug Therapy in HIV Infection

Glasgow, UK. 9-13 November 2008

Published: 10 November 2008

Journal of the International AIDS Society 2008, I I (SuppI I):O37 doi:I0.II86/I758-2652-II-SI-O37

This abstract is available from: http://www.jiasociety.org/content/I I/SI/O37

(c) 2008 Smith et al; licensee BioMed Central Ltd.

\section{Background}

Although much research has investigated treatment discontinuation and virologic failure (VF) to first-line ART regimens, less is known about second-line responses.

\section{Methods}

We included patients at the Royal Free Hospital, London, who were ARV-naïve when starting their first ART regimen ( $>=3$ ARVs) and who: (i) experienced VF to their first ART regimen (at least one viral load $>400 \mathrm{cps} / \mathrm{ml}$ after $>4$ months continuous exposure to an ARV); (ii) started second-line ART (defined by the first date on which at least one PI/NNRTI and/or at least two new NRTIs were started after VF to the first-line regimen occurred) with a latest viral load $>400 \mathrm{cps} / \mathrm{ml}$. Time to VF (the first of two consecutive viral loads $>400 \mathrm{cps} / \mathrm{ml}$ more than 4 months after starting their second-line regimen; ARV changes/discontinuations ignored) was calculated using survival analysis.

\section{Summary of results}

166 patients started second-line ART with a median (IQR) CD4 count and viral load of $256(120-358)$ cells/mm3 and $4.3(3.3-5.0) \log \mathrm{cps} / \mathrm{ml}$. Twenty-five (15\%), 48 $(29 \%)$ and $93(56 \%)$ patients second-line regimen included one, two and $>=3$ antiretrovirals they had not previously received. The median $(95 \% \mathrm{CI})$ time to discontinuing at least one ARV was 15 (12-19) months, and 24 (17-41) months for discontinuing a PI/NNRTI. Few factors were associated with time to first discontinuation of any ARV: compared to those starting a second-line regi- men containing one new ARV, the adjusted hazard ratios were $0.56(0.33-0.95)$ and $0.50(0.30,0.83 ; \mathrm{p}=0.001)$ for those receiving two and $>=3$ new ARVs. Each additional year in time from first VF to start of second-line regimen was associated with a $19 \%$ reduced hazard of discontinuing (0.66-0.99; $\mathrm{p}=0.04)$. 29\% (22-36\%) and 44\% (36$52 \%$ ) experienced VF by 12 and 36 months after starting second-line cART. This compares to $14 \%$ and $27 \%$ amongst those on first-line cART. The only factors associated with VF in multivariable analysis were the inclusion of fewer new ARVs in the second-line regimen (compared to one: $\mathrm{HR}=0.1 ; 0.13-0.75$ for two, and $0.26 ; 0.12-0.60$; $\mathrm{p}=0.01$ for $>=3$ new ARVs), lower CD4 count $(\mathrm{HR}=0.73$ per 100 cells $/ \mathrm{mm} 3 ; 0.59-0.96 ; \mathrm{p}=0.03)$, and higher viral load at the start of the second-line regimen $(\mathrm{HR}=2.56 \mathrm{per}$ $1 \log \mathrm{cps} / \mathrm{ml} ; 1.33-4.94 ; \mathrm{p}=0.005)$.

\section{Conclusion}

The median time to making at least one ARV switch on a second-line regimen was comparable to that seen on firstline regimens. Although VF appeared more common on second-line than on first-line regimens, perhaps because this is a group who are more predisposed to $\mathrm{VF}$, response rates were still excellent. 